\title{
Is Higher Viral Load in SARS-CoV-2 Associated with Death?
}

\author{
Klinger Soares Faíco-Filho, Victor Cabelho Passarelli, ${ }^{\star}$ and Nancy Bellei \\ Universidade Federal de São Paulo (UNIFESP), Laboratório de Virologia, Division of Infectious Diseases, Department of Medicine, Escola Paulista \\ de Medicina (EPM), São Paulo, Brazil
}

\begin{abstract}
There is no proven prognostic marker for patients hospitalized with COVID-19. We conducted a retrospective cohort study of patients hospitalized with COVID-19 from March 14, 2020 to June 17, 2020, at São Paulo Hospital, in São Paulo, Brazil. SARS-CoV-2 viral load was assessed using the cycle threshold (Ct) values obtained from a reverse transcription-PCR assay applied to the nasopharyngeal swab samples. The reactions were performed following the CDC U.S. protocol targeting the N1 and N2 sequences of the SARS-CoV-2 nucleoprotein gene and human ribonuclease $\mathrm{P}$ gene serving as an endogenous control. Disease severity and patient outcomes were compared. Among 875 patients, 50.1\% (439/875) were categorized as having mild disease (nonhospitalized patients), 30.4\% (266/875) moderate (hospitalized in the ward), and 19.5\% (170/875) severe disease (admitted to the intensive care unit). A Ct value of $<25$ (472/ 875) indicated a high viral load, which was independently associated with mortality (odds ratio [OR]: $2.93 ; 95 \% \mathrm{Cl}$ : 1.87-4.60; $P<0.0001$ ). We concluded that admission SARS-CoV-2 viral load was independently associated with mortality among patients hospitalized with COVID-19.
\end{abstract}

\section{INTRODUCTION}

The pandemic of SARS-CoV-2 has been responsible for causing more than 30 million infections around the world since December 2019. ${ }^{1}$ Although the severity is mild in most cases, several risk factors for moderate to severe COVID-19 have been identified, including older age and comorbidities. ${ }^{2,3}$ However, the association between SARS-CoV-2 viral load and clinical outcomes remains understudied. Two Chinese studies demonstrated that the viral load of hospitalized patients was higher than that of nonhospitalized ones. ${ }^{4,5}$ Also, a recent American study demonstrated that higher viral loads were associated with a higher risk of intubation and death for inpatients. ${ }^{6}$ Nevertheless, correlation between viral load and hospitalization risk for inpatients and outpatients remains unclear.

Detection of SARS-CoV-2 by the reverse transcription-PCR (RT-PCR) assay in nasopharyngeal swab specimens is the primary diagnostic method for COVID-19. ${ }^{7}$ Data concerning cycle threshold (Ct) values, which are inversely proportional to the amount of RNA virus' copies, have been used as an inference of the viral load. Based on the hypothesis that the SARS-CoV-2 Ct value could also act as a biomarker of disease severity, clinical outcome, and mortality, we conducted a retrospective analysis of 875 patients diagnosed with COVID19 at a large university hospital in São Paulo, Brazil, between March 2020 and June 2020.

\section{METHODS}

Study population and setting. This retrospective observational cohort study involved patients and healthcare workers (HCW) who presented with respiratory symptoms from March 14, 2020 to June 17, 2020, at São Paulo Hospital in the Federal University of São Paulo, Brazil. All hospitalized patients considered suspected of COVID-19 had a

\footnotetext{
*Address correspondence to Victor Cabelho Passarelli, Division of Infectious Diseases, Department of Medicine, Laboratório de Virologia, Escola Paulista de Medicina, Universidade Federal de São Paulo (UNIFESP), Rua Pedro de Toledo, 781 - Vila Clementino, São Paulo 04039-032, Brazil. E-mail: vicpassarelli@hotmail.com
}

nasopharyngeal swab sample collected on admission and analyzed for SARS-CoV-2. We also included all HCWs presenting with clinical suspicion of COVID-19 as part of a routine surveillance. All the specimens were stored in $2 \mathrm{~mL}$ sterile Ringer's lactate solution (Fresenius, Bad Homburg vor der Höhe, Germany) in sterile tubes and transported to the Virology Laboratory at the Federal University of São Paulo for testing.

Viral load analysis method. We used the Ct values as a semi-quantitative measure of viral load. The amount of viral RNA copies present in the positive samples is inversely proportional to the corresponding Ct value. That is, the greater the amount of viral RNA, the lower the Ct value.

Samples and RNA preparation. Nasal swab samples were collected from the patients at admission. RNA of the samples was purified using the Quick-RNA Viral Kit (Zymo Research, Irvine, CA), according to the manufacturer's instructions. The purified RNA was stored at $-80^{\circ} \mathrm{C}$.

SARS-CoV-2 detection. Viral detection was performed using the AgPath-ID One-Step RT-PCR reagents (ThermoFisher Scientific, Austin, TX), according to the manufacturer's instructions. The reactions were performed in a $20-\mu \mathrm{L}$ total reaction volume containing $5.0 \mu \mathrm{L}$ purified RNA, $400 \mathrm{nM}$ primers, and $200 \mathrm{nM}$ probes following the CDC U.S. protocol targeting the N1 and N2 sequences of the SARS-CoV2 nucleoprotein gene and human ribonuclease $P$ gene serving as an endogenous control. ${ }^{8}$ For the semi-quantitative analysis, we used the Ct values of the more sensitive N2 target. Samples with Ct values $<40$ were considered positive.

Data collection. Information on age, hospitalization status, and clinical outcome was retrieved from the electronic medical records of the patients. According to the WHO guidelines, patients were stratified into groups on the basis of age, as follows: 0-5, 5-14, 15-24, 25-34, 35-44, 45-54, 55-64, $65-74,75-84$, and $>85$ years. Disease severity and clinical outcome were classified as follows: mild (no hospitalization), moderate (hospitalization in the ward for observation and oxygen therapy), severe (hospitalization in the intensive care unit), and discharge or death. Patients who were still hospitalized, awaiting a defined outcome, during data collection were not considered for the statistical analysis. 
Statistical analysis. Statistical analyses were performed using the Student's $t$-test for parametric data and the Mann-Whitney test for nonparametric data. Significance level was set at a $P$ value of $<0.05$.

\section{RESULTS}

Among the 875 individuals with laboratory-confirmed COVID-19, more than a half $(50.9 \%, 446 / 875)$ were female patients. The median age was 48 years (range, 2-97 years), and the median Ct value was 24. A Ct value of $<25(472 / 875)$ indicated high viral load and $>24$ (403/875) low viral load.

The initial $\mathrm{Ct}$ values for each age-group are presented in Figure 1. We found no significant differences.

The Ct values were analyzed according to the disease severitymild (50.1\%; 439/875; median, 22); moderate (30.4\%; 266/875; median, 27), and severe (19.5\%; 170/875; median, 21.5)—in the 875 patients. The initial Ct value of patients with moderate disease was higher and significantly different from that of patients with mild $(P<0.0001)$ and severe $(P<0.0001)$ diseases (Figure 2).

Comparison of the initial Ct value with clinical outcome (discharge or death) showed that survivors presented a significantly higher value than that of non-survivors: median $\mathrm{Ct}$ values were 27 and 21, respectively $(P<0.0001$; Figure 3$)$. Mortality rates were $46 \%(87 / 191)$ among patients with a high viral load $(\mathrm{Ct}<25)$ and $22 \%(41 / 185)$ among patients with a low viral load. The risk of in-hospital mortality was also higher in patients with a high viral load $(\mathrm{Ct}<25)$ than in those with a low viral load (Ct $>24)$, and this association was statistically significant (OR: 2.93; 95\% Cl: 1.87-4.60; $P<0.0001$ ).

\section{DISCUSSION}

In this study, a higher admission SARS-CoV-2 viral load (Ct values $<25$ ) was associated with an outcome of death, as also demonstrated previously. ${ }^{6}$ The correlation of viral load with clinical outcome and disease severity has been investigated with different endemic respiratory viruses, but most results have not been conclusive. ${ }^{9-12}$ However, in the case of prepandemic avian influenza $\mathrm{H} 5 \mathrm{~N} 1$, higher viral loads were associated with more severe disease and poorer outcome, ${ }^{13}$ as seen with SARS-CoV-2 in our study.

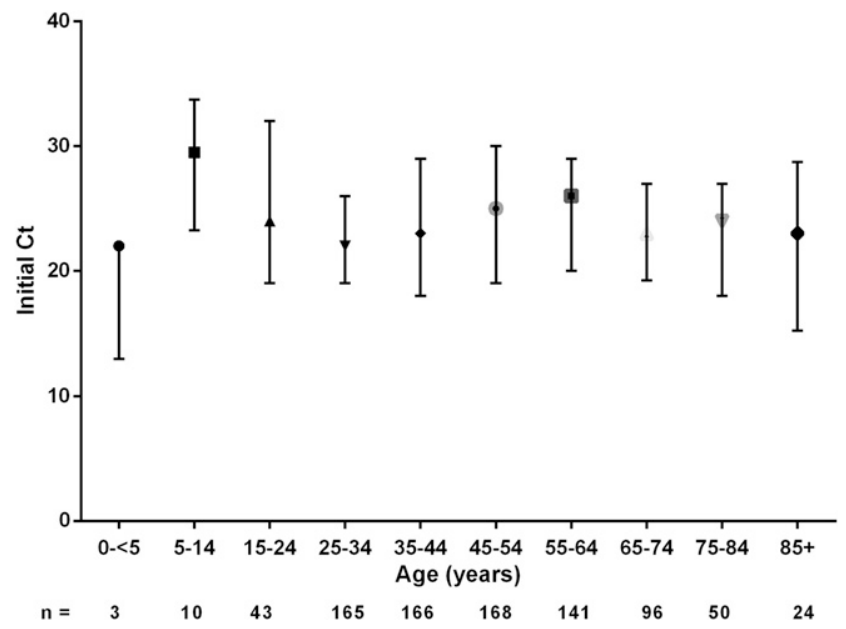

FIGURE 1. Initial cycle threshold (Ct) values from the swab samples in different age-groups.

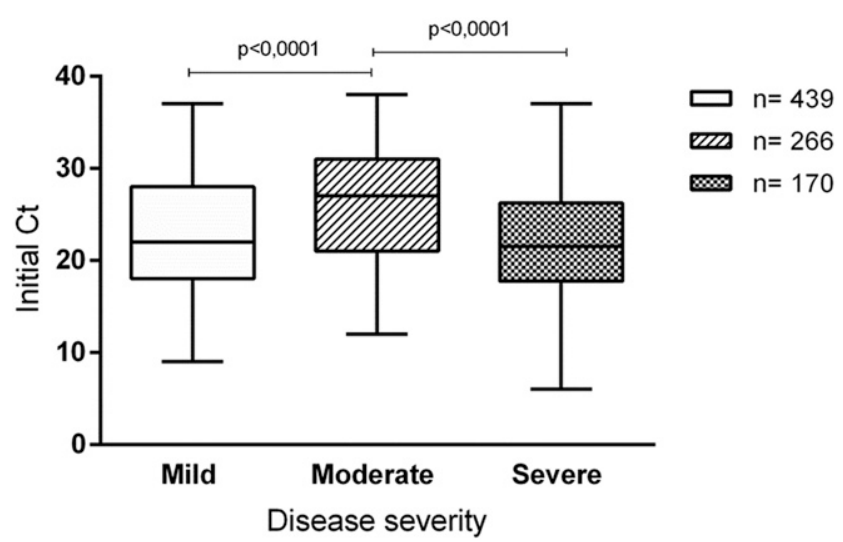

FIGURE 2. Initial cycle threshold (Ct) values from the swab samples obtained from patients with different clinical outcomes.

Notably, SARS-CoV-2 viral load distribution according to the $\mathrm{Ct}$ values from initial samples followed a $\mathrm{V}$ curve in this study, with mildly affected and critically ill patients having higher viral loads than the hospitalized ones with a better outcome. First of all, the population with mild disease in this study mainly included healthcare workers tested in a very early stage of disease, when viral replication is very high. ${ }^{4,5}$ Furthermore, high viral loads in patients with mild disease were also described by Argyropoulos et al. ${ }^{14}$ However, although their study demonstrated that hospitalized patients had a lower viral load than nonhospitalized ones, disease severity was not categorized.

On the other hand, our study presented a unique stratified clinical expression of disease including mild (nonhospitalized), moderate, and severe disease in the hospitalized. So the difference in viral loads between moderately affected and critically ill patients might be explained by later viral peak and longer virus-shedding periods in the latter. ${ }^{4,5}$ This could also be a result of orientation to complete isolation at home if no warning signs were present, to avoid emergency rooms' overcrowding. Therefore, most patients presented to the emergency department during a later period of disease, with more severe symptoms' onset.

The results of this study must be interpreted considering the methodological limitations. We focused on virological aspects and did not include the effects of comorbidities, clinical

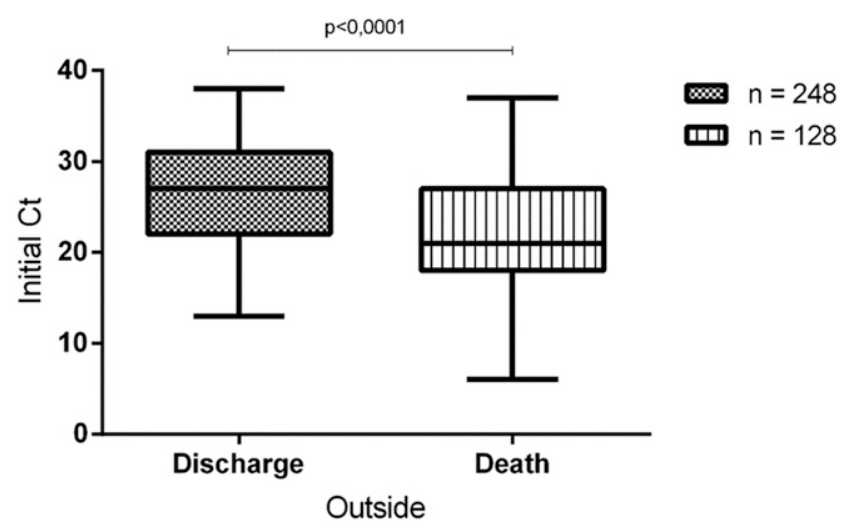

FIGURE 3. Cycle threshold (Ct) values from the swab samples according to discharge or death. 
symptoms, date of admission and sample collection, and use of antivirals or antibiotics because of the heterogeneity in the care of hospitalized patients, and requirement of a larger cohort for subgroup analysis. The duration of symptoms before testing may be an important variable. However, date of symptom onset was not consistently reported by medical assistants at this hospital. In any case, previous studies demonstrated that patients with other acute viral infections tend to present to the hospital after expected peak of viral load. ${ }^{15}$

In summary, admission SARS-CoV-2 viral load, demonstrated by the Ct value, was independently associated with mortality among patients hospitalized with COVID-19. These findings suggest that the Ct value could be used as a tool to help with the identification of patients at a higher risk for severe outcomes.

Received August 4, 2020. Accepted for publication September 21, 2020.

Published online September 28, 2020.

Acknowledgment: Publication charges for this article were waived due to the ongoing pandemic of COVID-19.

Authors' addresses: Klinger Soares Faíco-Filho, Victor Cabelho Passarelli, and Nancy Bellei, Division of Infectious Diseases, Department of Medicine, Laboratório de Virologia, Escola Paulista de Medicina, Universidade Federal de São Paulo (UNIFESP), São Paulo, Brazil, E-mails: klingerfaiko@hotmail.com, vicpassarelli@hotmail.com, and nbellei@uol.com.br.

This is an open-access article distributed under the terms of the Creative Commons Attribution (CC-BY) License, which permits unrestricted use, distribution, and reproduction in any medium, provided the original author and source are credited.

\section{REFERENCES}

1. Acter T, Uddin N, Das J, Akhter A, Choudhury TR, Kim S, 2020. Evolution of severe acute respiratory syndrome coronavirus 2 (SARS-CoV-2) as coronavirus disease 2019 (COVID-19) pandemic: a global health emergency. Sci Total Environ 730: 138996.

2. Richardson S et al., 2020. Presenting characteristics, comorbidities, and outcomes among 5700 patients hospitalized with COVID-19 in the New York city area. JAMA 323: 2052-2059.
3. Wang D et al., 2020. Clinical characteristics of 138 hospitalized patients with 2019 novel coronavirus-infected pneumonia in Wuhan, China. JAMA 323: 1061-1069.

4. Zheng $S$ et al., 2020. Viral load dynamics and disease severity in patients with SARSCoV-2 in Zhejiang province, China January-March 2020: retrospective cohort study. BMJ 369: $\mathrm{m} 1443$.

5. Liu Y, Yan LM, Wan L, Xiang TX, Le A, Liu JM, Peiris M, Poon LLM, Zhang W, 2020. Viral dynamics in mild and severe cases of COVID-19. Lancet Infect Dis 20: 656-657.

6. Magleby R, Westblade L, Trzebucki A, Simon MS, Rajan M, Park J, Goyal P, Safford MM, Satlin MJ, 2020. Impact of SARS-CoV-2 viral load on risk of intubation and mortality among hospitalized patients with coronavirus disease 2019. Clin Infect Dis ciaa851 (Epub ahead of print). Available at: https://doi.org/10.1093/cid/ ciaa851.

7. WHO, 2020. WHO Laboratory Testing for 2019 Novel Coronavirus (2019-nCoV) in Suspected Human Cases. Interim Guidance. Geneva, Switzerland: World Health Organization.

8. CDC, 2020. CDC 2019-Novel Coronavirus (2019-nCoV) Real-Time RT-PCR Diagnostic Panel. Available at: https://www.fda.gov/ media/134922/download. Accessed June 14, 2020.

9. Feikin DR et al., 2017. Is higher viral load in the upper respiratory tract associated with severe pneumonia? Findings from the PERCH study. Clin Infect Dis 64 (Supp/ 3): S337-S346.

10. Kadji FM et al., 2016. Differences in viral load among human respiratory syncytial virus genotypes in hospitalized children with severe acute respiratory infections in the Philippines. Virol J 13: 113.

11. Granados A, Peci A, McGeer A, Gubbay JB, 2017. Influenza and rhinovirus viral load and disease severity in upper respiratory tract infections. J Clin Virol 86: 14-19.

12. Lalueza A et al., 2019. Influence of viral load in the outcome of hospitalized patients with influenza virus infection. Eur J Clin Microbiol Infect Dis 38: 667-673.

13. Pawestri HA, Eggink D, Isfandari S, Thanh TT, Rogier van Doorn $\mathrm{H}$, Setiawaty V, de Jong MD, 2020. Viral factors associated with the high mortality related to human infections with clade 2.1 influenza $\mathrm{A} / \mathrm{H} 5 \mathrm{~N} 1$ virus in Indonesia. Clin Infect Dis 70: 1139-1146.

14. Argyropoulos KV et al., 2020. Association of initial viral load in SARS-CoV-2 patients with outcome and symptoms. Am J Pathol 190: 1881-1887.

15. Hadjichrysanthou $C$, Cauët $E$, Lawrence $E$, Vegvari $C$, De Wolf $F$, Anderson RM, 2016. Understanding the within-host dynamics of influenza A virus: from theory to clinical implications. $J R$ Soc Interf 13: 20160289. 